\section{Shoe Covers and Tacky Mats}

\section{To the Editor:}

I have read in several journals and heard in a seminar of the ineffectiveness of shoe covers and tacky mats used in operating room suites. The staff strongly objects to the discontinuation of these "protective" measures. It is true that shoe covers do protect shoes and lessen the time to clean them, but it seems like we pay a high bill for shoe cleaning. But, for infection control?

Is there any recent information that would justify continuing their use? To discontinue them will mean a considerable savings, which of course speaks loudly these days.

Your assistance will be appreciated.

Lenna Lee Davidson, RN, MPH Infection Control Nurse Ardmore Adventist Medical Center Ardmore, Oklahoma

Ms. Sue Crow, nurse epidemiologist, responds to Ms. Davidson's letter:

Tacky mats have no place in hospitals or skilled care facilities. They only provide a false sense of security. Actually, you can pick up as much dirt from one that is soiled as you put down. You can easily cut costs in health care facilities by eliminating these expensive mats.

Shoe covers are a controversial subject in today's hospital. Many recommending organizations now do not feel that shoe covers affect the nosocomial infection rate, and consequently, do not advocate using shoe covers. Before you discontinue them, however, consider that the OR should be kept as clean as possible and that clean shoes promote this concept. If for some obscure reason the OR team wears dirty shoes or if they never clean the shoes they wear solely in the OR, shoe covers may help keep the OR cleaner. Look at your individual concerns, use a little common sense, and then decide what's best for your situation.

Sue Crow, RN, MSN, CIC Nurse Epidemiologist Louisiana State University Medical School Shreveport, Louisiana

\section{Infection Indicated by Urinary Odor}

\section{To the Editor:}

In the article "When Should We Use Urine Cultures?" by Walter E. Stamm, MD (Infect Control 1986; 7[August]: 431-433), Dr. Stamm includes urinary odor as a symptom or sign of possible infection in a catheterized patient, indicating the need for a urine culture. I would like to ask Dr. Stamm what scientific evidence he has to support that statement.

Sandra L. Loss, RN Director of Infection Control Niagara Falls Memorial Medical Center Niagara Falls, New York
$D r$. Stamm responds to Ms. Loss' letter:

I am not aware of a specific study that has addressed this issue, and to my knowledge the sensitivity and specificity of urinary odor as an indicator of infection are unknown. However, patients and clinicians alike have often observed this association, and thus the statement is supported largely by clinical experience.

Walter E. Stamm, MD Head, Division of Infectious Diseases Harborview Medical Center Seattle, Washington

Editorial correction: Dennis L. Murray, MD, whose letter to the editor appeared in the February issue (Infect Control 1987; 2:48) was mistakenly identified as being from Michigan State University in Ann Arbor. Michigan State University is located in East Lansing. The editor regrets any inconvenience causd to our readers by this error.

Letters to the Editor should be addressed to INFECTION CONTROL Editorial Offices, C41 General Hospital, University of Iowa Hospitals and Clinics, Iowa City, IA 52242. All letters must be typed, double-spaced, and may not exceed four pages nor include more than one figure or table. The editors reserve the right to edit for purposes of clarity or brevity. 


\section{POST-}

\section{The Ultimate In-Room Sharps Containment System}

The Answer to your Sharps Disposal Problem-an in room system with a vertical drop. The proven effective, efficient way to avoid sticks.

- Lockable, metal cabinets and plastic containers are available in both six and ten quart sizes to meet your specific needs.

- Sharps disposal with an easy, natural hand motion.

re

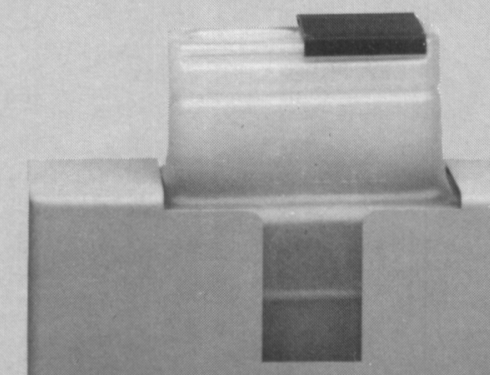

(6)

Pat. Pend.

\section{(e)}

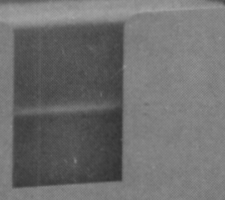

\section{Don't Evaluate Without POST!}

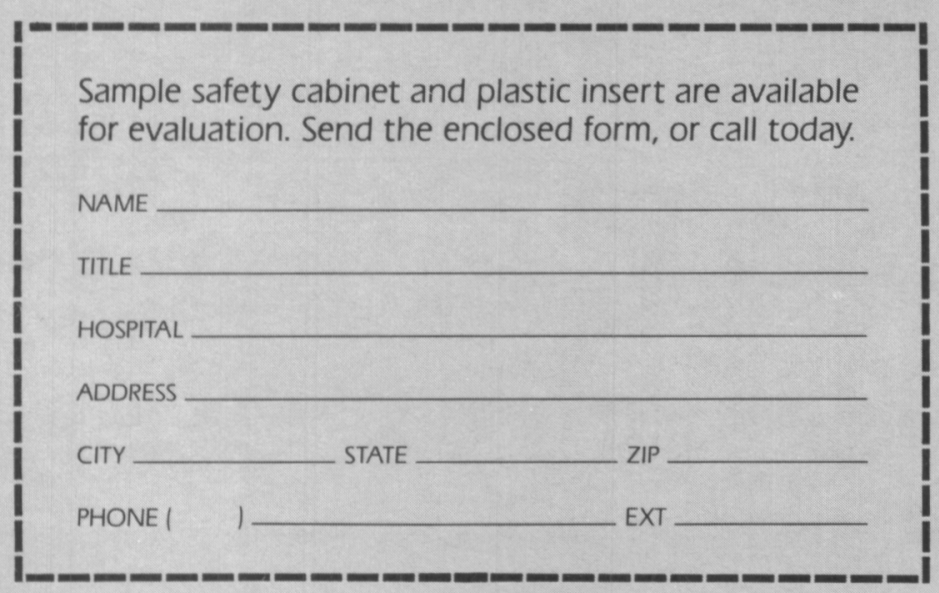

- Sharps FILL LEVEL is always in view, controlling over filling.

- Safe, immediate disposal of large size needles and syringes. 